\title{
Human-dog relationships during the COVID-19 pandemic: booming dog adoption during social isolation
}

\author{
Liat Morgan (iD) et al."
}

The recent COVID-19 pandemic led to uncertainty and severe health and economic concerns. Previous studies indicated that owning a companion animal, such as a dog or a cat, has benefits for good mental health. Interactions with animals may help with depression and anxiety, particularly under stress-prone conditions. Human-animal interactions may even improve peer-to-peer social relationships, as well as enhance feelings of respect, trust, and empathy between people. Interestingly, it has also been shown that stress and poor wellbeing of dog owners negatively affect the well-being of their companion animals. However, a dramatic increase in dog abandonment could potentially occur due to COVID-19 related health, economic and social stresses, as well as due to the inconclusive reports of companion animals being potential COVID-19 carriers. Such a scenario may lead to high costs and considerable public health risks. Accordingly, we hypothesized that the COVID-19 pandemic, and the related social isolation, might lead to dramatic changes in human-dog bidirectional relationships. Using unique prospective and retrospective datasets, our objectives were to investigate how people perceived and acted during the COVID-19 pandemic social isolation, in regards to dog adoption and abandonment; and to examine the bidirectional relationship between the well-being of dog owners and that of their dogs. Overall, according to our analysis, as the social isolation became more stringent during the pandemic, the interest in dog adoption and the adoption rate increased significantly, while abandonment did not change. Moreover, there was a clear association between an individual's impaired quality of life and their perceptions of a parallel deterioration in the quality of life of their dogs and reports of new behavioral problems. As humans and dogs are both social animals, these findings suggest potential benefits of the human-dog relationships during the COVID-19 pandemic, in accordance with the One Welfare approach that implies that there is a bidirectional connection between the welfare and health of humans and non-human animals. As our climate continues to change, more disasters including pandemics will likely occur, highlighting the importance of research into crisis-driven changes in human-animal relationships.

\footnotetext{
\#A list of authors and their affiliations appears at the end of the paper.
} 


\section{Introduction}

he virus SARS-CoV-2 emerged in December 2019, in Wuhan, China. This unknown respiratory disease developed into the pandemic, termed COVID-19, as declared by the World Health Organization on March 2020 (Bojdani et al., 2020). One of the main approaches worldwide for combating the disease is social isolation and distancing, at least until a protective vaccine is available (Koo et al., 2020; Lewnard and Lo, 2020; Bavel et al., 2020). Social isolation may prevent the spread of the disease, but it may also lead to other concerns. One of the greatest concerns regarding the influence of social isolation is its psychological effect on humans. Extended social isolation may lead to a significant decrease in quality of life and well-being, and high levels of stress, in both the infected and non-infected populations (Xiao et al., 2020; Bavel et al., 2020). Social isolation is an additional stressor to an already highly stressful world environment and people's extensive fear of the novel COVID-19 pandemic threat (Bavel et al., 2020; LeDoux, 2012; Mobbs et al., 2015). In addition, social distancing included full lockdowns in many countries, as well as in Israel, with dramatic economic effects (Anser et al., 2020; Sangar et al., 2019). Adverse local and global economic impacts, in addition to drastic personal income reduction, may be detrimental to people's psychological health and general well-being (Xiao et al., 2020).

Interestingly, the mental health benefits of owning a companion animal, such as a dog or a cat, have been shown by several scientific studies (Serpell, 1991; Beetz et al., 2012; Powell et al., 2019). The majority of studies indicate that interactions with animals may help with depression, anxiety, and stress, in particular under stress-prone conditions (Beetz et al., 2012). On the one hand, companion animals provide companionship, improve mood, and may ease loneliness; human-animal interactions may even improve peer-to-peer social relationships, as well as enhance feelings of respect, trust, and empathy between people (Powell et al., 2018; Beetz et al., 2012; Powell et al., 2019). On the other hand, it has also been shown that stress and poor well-being of owners negatively affect the stress and well-being of their companion animals (Buttner et al., 2015; Sumegi et al., 2014; Ryan et al., 2019). For example, there has been some indication that the stress of the owner could influence their dog's cognitive ability (Sumegi et al., 2014). Moreover, changes in the attention of owners to their dogs may affect the behavior of the dogs (Kaminski et al., 2009; Payne et al., 2016). Therefore, we hypothesized that the COVID-19 pandemic might lead to dramatic changes in human-dog bidirectional relationships. On the one hand, owning a dog may assist the owner in coping with the stressful world situation, and therefore, more people may decide to adopt a dog during this pandemic. On the other hand, behavioral problems in dogs were reported to be one of the main reasons for the abandonment of dogs to shelters (Patronek et al., 1996; Salman et al., 2000); if changes in the lives of owners occurred during the COVID-19 pandemic, and indeed, if behavioral problems in their dogs developed as was shown under other circumstances (Sumegi et al., 2014), then this might increase the risk of dog relinquishment.

Another potential risk factor for dog abandonment and relinquishment during the COVID-19 pandemic was their suspected epidemiological role in the spread of SARS-CoV-2. There was a worldwide growing concern that companion animals, specifically dogs and cats, could transmit the disease to humans (Goumenou et al., 2020; Parry, 2020; Leroy et al., 2020). Although the anecdotal reports were inconclusive, it could lead to an increase in the number of dogs relinquished by their owners. Thus, overall, the inconclusive reports of companion animals being potential carriers of the COVID-19 virus, the economic crisis, and the general stress and panic during this pandemic, could potentially cause a dramatic increase in dog abandonment numbers. Since such a scenario might incur high costs and present considerable risk to public health, it should be explored. Relinquishment and abandonment of companion animals is a global problem. It is estimated that millions of pets are abandoned each year (Fatjo et al., 2015), even without a pandemic in the background. It results in increasing numbers of free-roaming animals, overcrowded animal shelters, impaired animal welfare, and it carries high costs to tax payers (Fatjo et al., 2015). Moreover, it is a severe public health issue due to the potential transmission of zoonotic diseases (such as rabies) and attacks on people (Carter, 1990; Burgos-Caceres, 2011). All of these threats also carry remarkable economic consequences, which affect national and local governments, humane organizations, as well as individuals (Carter, 1990).

In 2012, an online, searchable database of animals that need homes in Israel was established (http://Yad4.co.il), by the first author. The first and only project of its kind in Israel, Yad4, serves as a national database for dog adoption, as it includes the vast majority of abandoned dogs that need homes throughout the country. As such, the established database provides both an understanding of the current landscape of dog abandonment and adoption at any given moment, as well as a unique look into the longitudinal relationships of dogs and people as the same dogs may be tracked across time, multiple homes, and shelter stays. The Yad4 initiative aims to rescue abandoned animals in Israel by increasing adoption rates, reducing the extent of dog euthanasia, and shortening the length of stay at the shelters until adoption, and has no profit purposes. The website offers a user-friendly search engine for potential adopters to find available dogs from organizations and municipal shelters across the country of Israel. The information is uploaded and updated by animal welfare organizations and municipal veterinarians, typically as soon as they have the dog in their possession. As of 2020, 72 animal welfare organizations and municipal shelters are registered and active on the website, each managing its own pool of adoptable pets independently, with its own online account. During the COVID-19 pandemic, the website operated as usual; although, initially, there was a concern for massive abandonment and a decrease in adoption.

In order to control the pandemic, gradual social restrictions were initiated during March 2020 in Israel, while in April, a total lockdown was implemented for a full month by the Israeli government, as marked on the timeline in Fig. 1. During this period, walking the dog and veterinary care were exceptions for the lockdown restrictions, as well as dog adoptions from animal welfare organizations and municipal shelters. Therefore, whereas it was not allowed to be outside of a $100 \mathrm{~m}$ radius from your home, dog adoption and dog walking were permitted throughout these periods.

The objectives of this study were to investigate: (1) how the COVID-19 pandemic affected adoption and abandonment of dogs at shelters, and the public's general interest in adopting a dog; (2) the association between the quality of life of owners and their dogs during the pandemic; as well as (3) the effect of the pandemic on the development of new behavioral problems and on the relinquishment rate of dogs by their owners.

\section{Results}

This study focused on a new aspect of the COVID-19 pandemic by investigating the human-dog relationship during this crisis. Dog adoptions, abandonment, as well as the association between the well-being of the owners and their perceptions of the quality of life of their dogs, were examined. Overall, in contrast to some of the initial concerns, all dog adoption measures significantly 


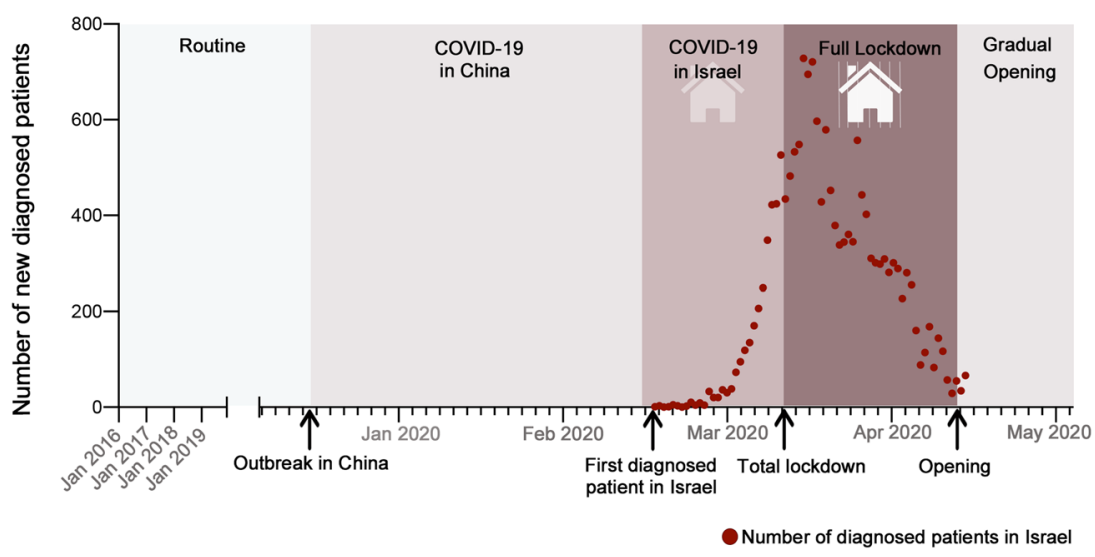

Fig. 1 Timeline of COVID-19 pandemic in Israel. The different colors, which get darker, represent the various periods analyzed in this study (x-axis): before the COVID-19 outbreak in China (years 2016-2019; light gray); from the initial outbreak in China until the first diagnosed patient in Israel (dark gray); during the outbreak in Israel, from the diagnosis of the first COVID-19 patient until lockdown declared by the Israeli government (light brown); during the full lockdown for a month (brown); and the gradual opening on May (gray, on the right side of the figure). The daily number of new diagnosed COVID-19 patients in Israel is represented as red dots.

improved as the social restrictions became stricter. Furthermore, there was a clear association between an individual's quality of life and their perceptions of their dog's quality of life and behavior, as well as the probability of their relinquishing their pet.

Changes in dog adoption and abandonment. The database of Yad4 website was analyzed in order to investigate dog abandonment and adoptions under the growing pressure of the COVID-19 pandemic. Most abandoned dogs which are offered for adoption in Israel are published on Yad4 website, which includes most animal welfare organizations and municipal shelters for dogs. Therefore, the dogs uploaded on a daily basis to the website represent the abandoned dog population, which includes mainly dogs that were relinquished by their owners. Overall, according to our analysis, the stricter the social restrictions became during the COVID-19 pandemic in Israel, the number of potential adopters (people looking to adopt a dog), as well as the dog adoption rate, increased significantly (Fig. 2); while dog abandonment did not change. Multiple linear regression analyses of Yad4 records from January 2016 to May 2020 revealed that the main periods during the development of the pandemic in Israel were significantly associated with dog adoption measures, while the abandonment rate did not change (Fig. 2; Supplementary Table S1). The number of dogs uploaded to the website, representing most of the abandoned dogs in Israel, did not change significantly over the years, including during the COVID-19 pandemic (Fig. 2a-c). On the contrary, adoption measures were significantly affected by the different periods (Fig. 2d-i), particularly after the first COVID-19 patient in Israel was diagnosed, and even to a further extent during the social lockdown. Between the time that the first patient in Israel was diagnosed to the full lockdown of the country, the average number of adoption requests submitted online was $31.1 \pm 1.9$ (Mean \pm SEM) requests per day; during the total lockdown, the average number of dog adoption requests was $111.3 \pm 4.1$ requests per day; and during the gradual opening on May, $73 \pm 4.6$ adoption requests were submitted per day. However, before the COVID-19 outbreak in China, the average daily number of dog adoption requests was only $25.7 \pm 4.1$ requests per day. Linear regression analysis revealed that after controlling for the effects of the month, the year and governmental initiatives for the encouragement of responsible dog ownership between 2018 and 2019, the increase in the number of adoption requests during the outbreak in Israel, and the full lockdown, were significantly higher than the period before the COVID-19 outbreak in China $(P<0.05$; Fig. 2d-f; Supplementary Table S1). Accordingly, the average number of adopted dogs increased significantly already following the outbreak in China, as well as during the outbreak in Israel and the full lockdown, as compared to before the pandemic $(P<0.05$; Fig. $2 \mathrm{~g}-\mathrm{i}$; Supplementary Table S1). Immediately after the outbreak in China, the average daily number of adopted dogs was $17.3 \pm 2.2$ dogs per day; during the outbreak in Israel it was $22.8 \pm 2.1$ adopted dogs per day; during the total lockdown it was $26.1 \pm 2.2$ adopted dogs per day; and after the gradual opening it was $14.7 \pm$ 1.1 adopted dogs per day, which is similar to the period before the COVID outbreak in China (14.1 \pm 0.3 adopted dogs per day). Furthermore, as compared to the years prior to the COVID-19 pandemic, the length of stay (LOS) of the dog at the shelter, calculated as the interval from the time the dog was uploaded online to the Yad4 website until it was marked by the organizations as adopted, was significantly shorter following the media report of the COVID-19 outbreak in China and subsequently, with the shortest LOS $(10.1 \pm 0.5$ days $)$ during the full lockdown. Potential effects such as the month, the year, and governmental initiatives were controlled in the linear regression models (Supplementary Fig. S1; Supplementary Table S1; $P<0.05$ ).

Another option available for the public on Yad4 website was to fill in a request to serve as a foster family, as an alternative to adoption. Usually, the demand for foster families among the organizations is very high, but the number of available foster families is low. Therefore, typically, there are no available foster families since the organizations use them all. During the pandemic period, the number of foster families was higher than the demand. Accordingly, from the reports about the outbreak in China until the end of the lockdown in Israel, as well as during the gradual opening, the number of available foster families increased significantly. For example, as described in Fig. 3a, b, by the end of April 2019 there were no available foster families on Yad4 website, since they were all occupied and used by the organizations; contrarily, at the time of the outbreak in China, 226 foster families were available but did not receive a dog to foster, and by the end of April 2020, there were 844 available foster families.

Local and global online searches for adoptable dogs. The daily number of visitors on the Yad4 website since the first COVID-19 patient diagnosed in Israel until the end of the full lockdown was significantly higher, as compared to the whole period before the 
a

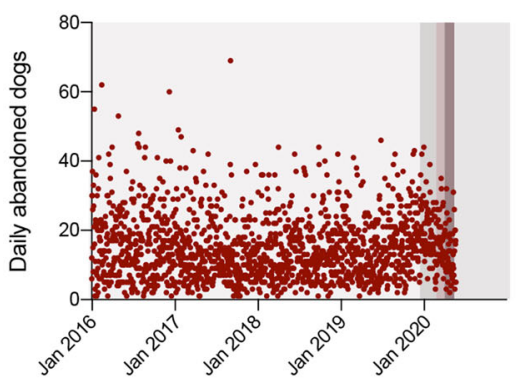

d

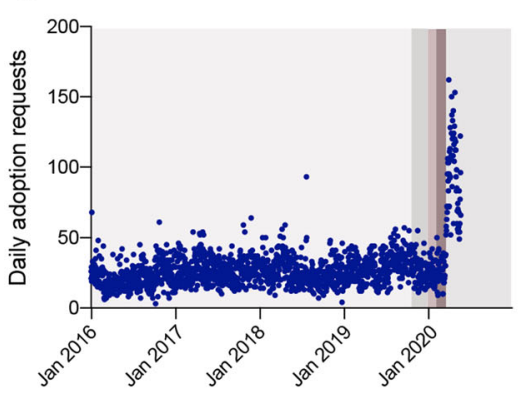

g

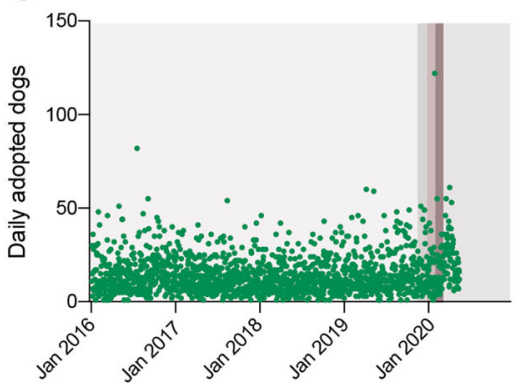

b

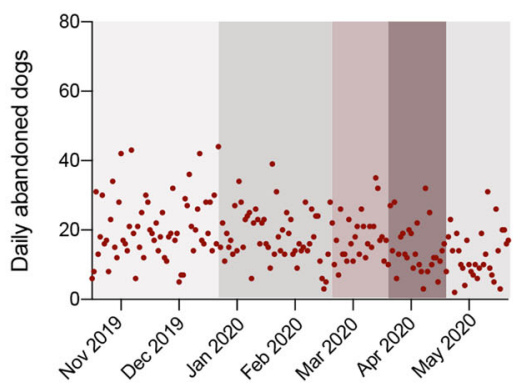

e

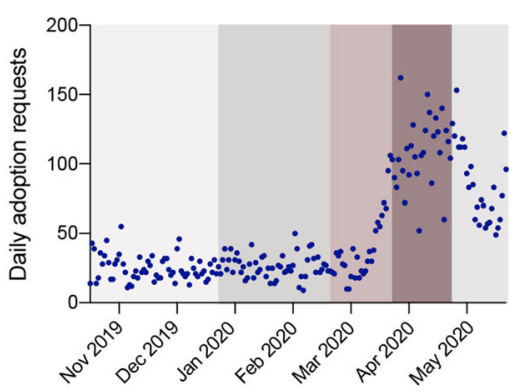

h

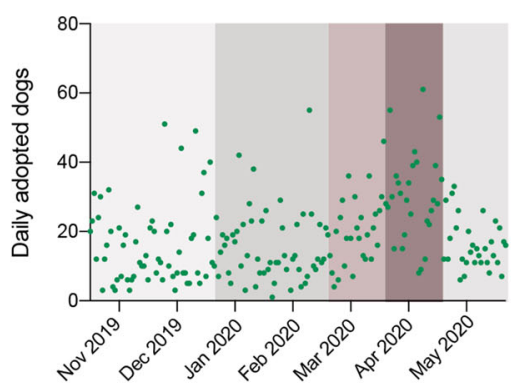

C

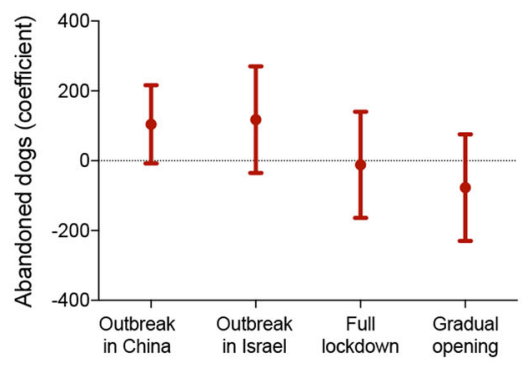

f

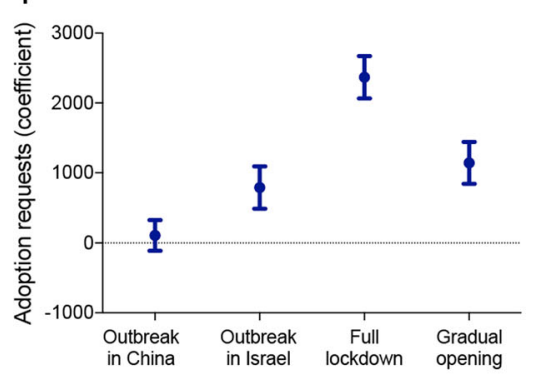

i

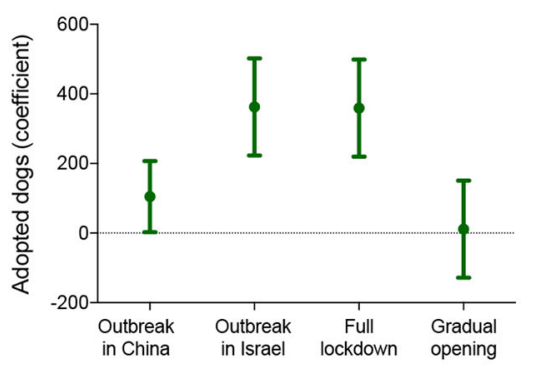

Fig. 2 Dogs' adoption and abandonment measures, during the COVID-19 outbreak in Israel. Each row represents data of a different variable: upper row (panels a-c) number of abandoned dogs (marked in red); middle row (panels $\mathbf{d - f}$ ) number of adoption requests made by potential owners (marked in blue); lower row (panels $\mathbf{g}-\mathbf{i}$ ) number of adopted dogs (marked in green). Daily data is presented on the first and second columns. Each dot represents the daily number of each parameter, to demonstrate trends over time. In the left column (panels $\mathbf{a}, \mathbf{d}, \mathbf{g}$ ), data are presented from 2016 until May 2020 . On the middle column (panels $\mathbf{b}, \mathbf{e}, \mathbf{h}$ ) data are presented as zoom-in, from November 2019 to May 2020. Period of times related to the COVID-19 pandemic are separated by colors, as detailed in Fig. 1. In the right column (panels $\mathbf{c}, \mathbf{f}, \mathbf{i}$ ), the results of Multivariate Linear Regression models are presented. In these models, the predictors were: the different time periods, from the outbreak in China to outbreak in Israel, the developments in Israel until full lockdown, full lockdown, and gradual opening; each period was compared to the period prior to COIVD-19 pandemic (from 2016 until the outbreak in China, represented by the horizontal dotted line); controlled for year, month, and governmental initiatives for dog adoption on 2019. The data are presented as coefficients (large dots) and its $95 \%$ confidence interval (bars); $P<0.05$.

pandemic (Fig. 3). The effect of year and month were controlled in the models (Supplementary Table S1). The linear regression model revealed that there was a significant increase in daily visits online when the outbreak emerged in Israel during March by $657.9 \pm 80.8$ (coefficient \pm SE) visits, and by $2311 \pm 82.1$ daily visitors online during the total lockdown period (Fig. 3a-c; $P<$ 0.05). For example, the absolute number of visits online in April 2020 was 221,959 visits, as compared to 72,703 in April 2019, and 91,920 visits in October 2019, which is typically the busiest season of the website. Interestingly, according to global non-scientific media reports, the demand for adoptable dogs worldwide was also high in other countries. Pictures of empty cages from many countries were published, but until now, to the best of the authors' knowledge, no scientific data has yet been published documenting this phenomenon. Thus, the global trend was investigated by analyzing Google Trends data for searches all around the world, as well as specifically in the USA. In order to do so, the timeline was divided to three periods: (1) before the outbreak in China; (2) from the first media reports about the outbreak in China on December 27th until March 13th-when the World Health Organization (WHO) announced Europe to be the epicenter of the pandemic; (3) the main lockdown worldwide -from the announcement of the WHO until the gradual opening on May; and (4) during May. The effect of year and month were controlled in the models (Supplementary Table S2). Interestingly, the world trends, according to the Google Trends data, were found to be similar to that we report herein for Israel (Fig. 3). The trends of worldwide searches online for "adopt a dog" were 
a

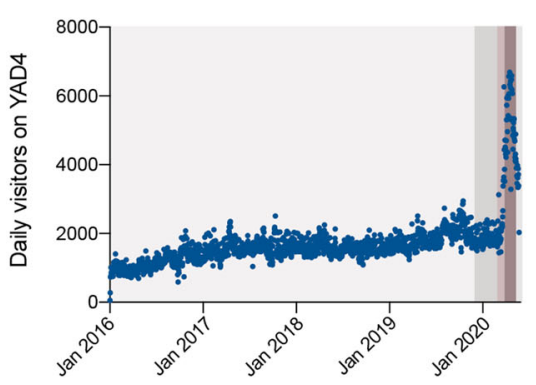

d

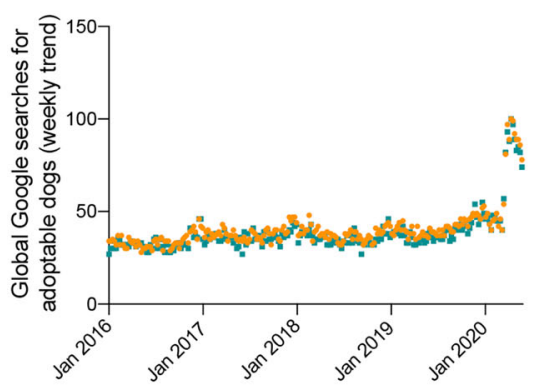

b

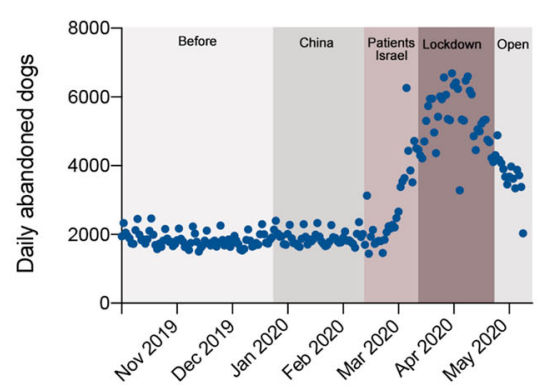

e

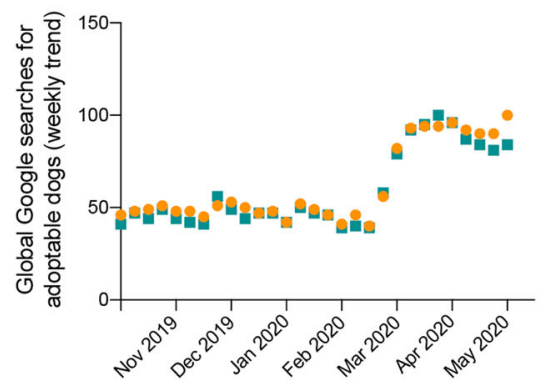

c

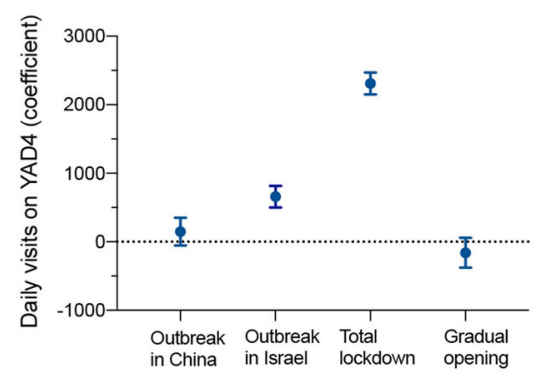

Worldwide Searches

f

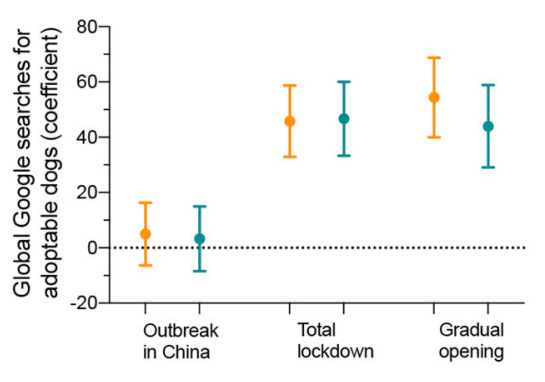

Fig. 3 Online users' visits in the Israeli Yad4 website, and worldwide Google searches, for adoptable dogs before and during COVID-19 pandemic. a The daily numbers of visitors on Yad4.co.il, the Israeli adoption search engine, from January 2016 to May 2020. b Zoom-in on the same data as in panel a during COVID-19 pandemic, from November 2019 to May 2020. c Results of the Linear Regressions model for Yad4 online visits, in each period during the COVID-19 pandemic, as compared to before the pandemic. In these models, the predictors were: the different periods, from the outbreak in China to outbreak in Israel, the developments in Israel until full lockdown, full lockdown, and gradual opening; each period was compared to the period prior to the COVID-19 pandemic (from 2016 until the outbreak in China, represented by the horizontal dotted line); controlled for: year, month, and governmental initiatives for dog adoption on 2019. d The weekly trends of Google searches for "adopt a dog" are presented from November 2019. e Zoom-in on the same data as in panel d, during COVID-19 pandemic. Both worldwide searches (orange) and USA searches (blue) are presented. f Results of the Linear Regressions model for global searches for adoptable dogs. In this model, the predictors were: the different periods, from the outbreak in China to the declaration of the World Health Organization on Europe as the epicenter of the pandemic, during the time most of the world was under restricted social isolation, and the gradual opening on May 2020. Each period was compared to the period from January 2019 to the outbreak in China (represented by the horizontal dotted line); controlled for: year and month. In panels c and f, data are presented as coefficients (large dots) and its $95 \%$ confidence interval (bars); $P<0.05$.

significantly higher during the periods of the outbreak in China, as well as during the period many countries declared lockdowns, as compared to the year of 2019 (Fig. 3d-f).

Given the high demand for dogs to adopt during the pandemic, the second part of our study included questionnaires targeting people who had recently adopted a dog, as well as current general dog owners, to explore the motivation behind this increase in demand for adoptable dogs.

The motivation for dog adoptions during COVID-19 pandemic lockdown, and the return rate of dogs back to shelters after the gradual opening of the lockdown. An online questionnaire was carried out in order to explore the reasons for dog adoption, particularly during the COVID-19 related lockdown, as well as to explore the return rate of the adopted dogs to the shelters, during the lockdown, and after the opening of the lockdown. This questionnaire was active for five days, starting on May 20th, 2020 (20 days after the gradual opening of the lockdown), and targeted people who adopted a dog from a shelter during the COVID-19 pandemic. The questionnaire targeted individuals who had adopted a dog as described in the :Methods" section, resulting in $n=508$ people in total; 312 of the respondents stated that they had adopted a dog during the pandemic
(January-May). Of these 312 new dog owners, 38.5\% of participants stated they had considered adopting a dog for a long time, and being at home during the COVID-19 lockdown seemed like a good opportunity; $37.8 \%$ stated that they had planned to adopt a dog regardless of the situation; $8.0 \%$ stated they felt lonely and/or stressed and believed that owning a dog might help; $9.3 \%$ had heard about dog abandonment in the media and felt it was the right thing to do; and a few people adopted for other reasons, as detailed in Fig. 4. Only 8 of the participants, who had adopted a dog during the pandemic $(2.6 \%)$, had already returned or relinquished the dog or have been considering relinquishment.

The association between impaired quality of life of owners to their perception of the quality of life of their dogs. In order to study the association between the quality of life of owners and their companion dogs under the COVID-19 pandemic situation, a digital questionnaire for dog owners was active during the full lockdown and social isolation (April). Participants replied to questions regarding their own well-being, as well as the well-being of their companion dog. Questions such as the effect of the pandemic on their stress level and personal finances, their concern about their own health, and their perceptions regarding their dog's well-being and behavior under the COVID-19 related 


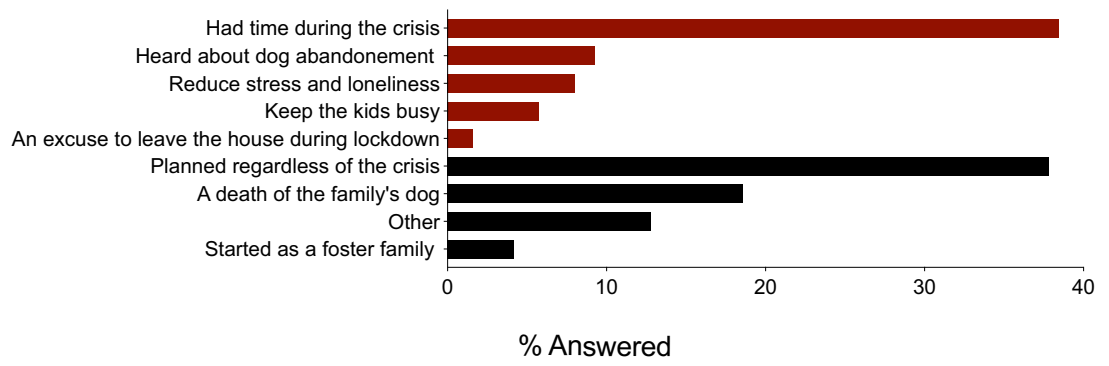

Fig. 4 Reasons for dog adoption during the COVID-19 pandemic. The frequencies of the participants' statements for the reason to adopt specifically during the pandemic are presented. Reasons related to the pandemic are marked in red. Other reasons are presented in black.

a Health concern

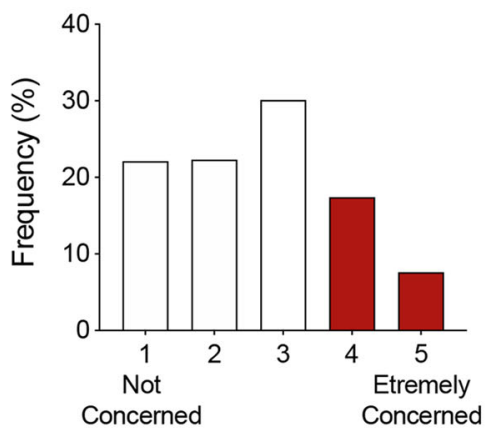

b Stress level

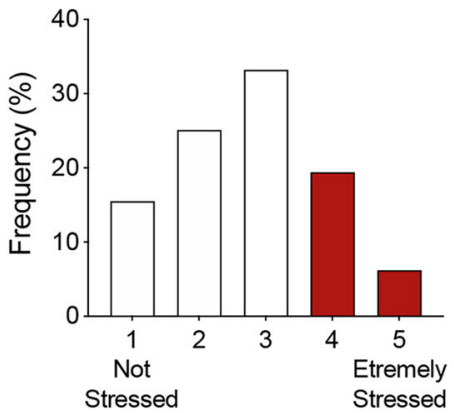

c Economic damage

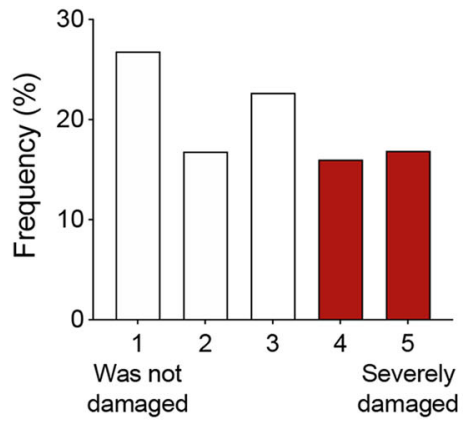

d Impaired quality of life of the owner index

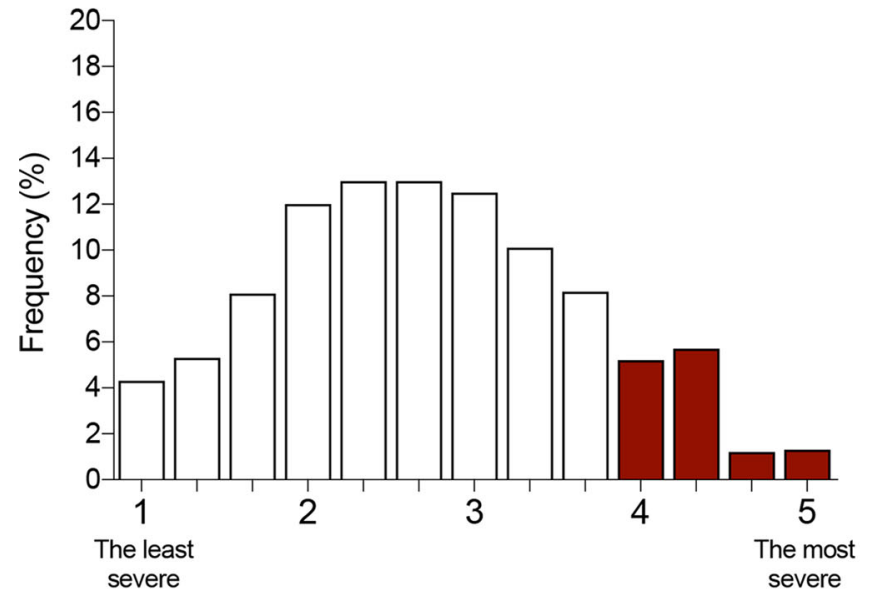

e

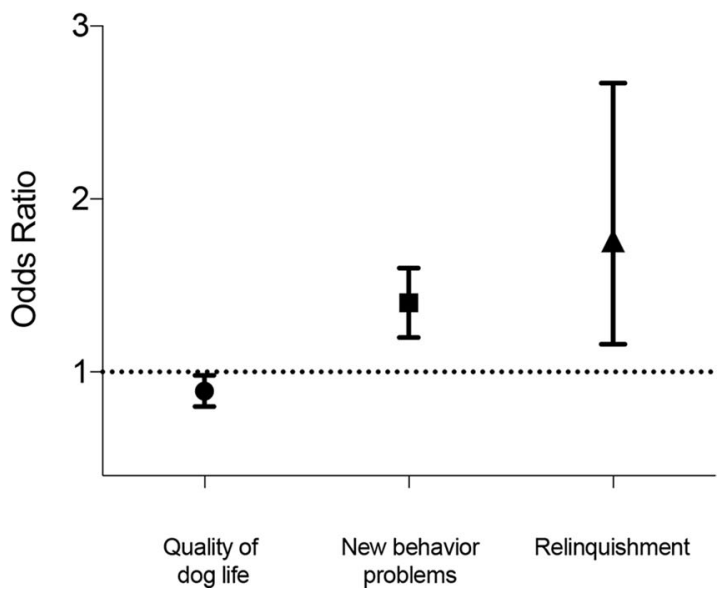

Fig. 5 Association between dog owners' life-quality and their perceptions of their dog's life-quality and behavior. a Frequencies of participants' answers regarding their concern for their own health on a scale of 1-5. b Frequencies of participants' answers regarding their stress level on a scale of 1-5. c Frequencies of participants' answers regarding their personal economic damage on a scale of 1-5. d Index of the impaired quality of life of the owner, based on the data presented in panels a-c. e Results of Logistic Regression model for dog's parameters by the increased impaired quality of life of the owners, as reported by the owner; quality of life of the dog (round dot), development of dog behavioral problems (square), and the intention of the owner to abandon the dog (triangular). Data are presented as odds ratios and its $95 \%$ confidence interval (bars); $P<0.05$ when the $95 \%$ confidence interval does not cross the horizontal dotted line.

lockdown, were included. The questionnaire also included questions regarding the characteristics of the owners and their dogs, as well as the care they provided to their dog during the pandemic. These variables were controlled in the statistical models (details in Supplementary Table S3). The outcome variables were set on a scale of 1-5 (for example, 1-low stress; 5-extremely stressed). Scores 4 and 5 were relabeled as "severe stress" for the analyses, and they were compared to scores 1-3: "none to moderate". The questionnaire was answered by $n=3138$ individuals. Overall, $25 \%$ of the participants were very concerned about their health
(Fig. 5a), 25.6\% stated they were extremely stressed (Fig. 5b), and $22.9 \%$ reported that their personal finances were severely affected (Fig. 5c). For further analysis, an impaired quality of life index was calculated as the mean of these scores (general stress; concern for their own health; and the damage to their personal financial situation; Fig. 5d). In addition, in the questionnaire, owners were asked to rank on a scale of 1-5 their assessment of the quality of life of their dogs during the COVID-19 lockdown, as well as their recognition of new behavioral problems, and whether they have considered relinquishing their dog. 
As hypothesized, multivariate logistic regressions revealed that an increase in the impaired quality of life index of the owner was associated with lower quality of life of the dog, as was assessed by the owner (odds ratio: 0.887 times lower for every one-unit increase in owner's quality of life index; Fig. 5e; $P<0.05)$. In addition, for a one-unit increase in impaired quality of life index of the owner, the odds ratio for recognition of new behavioral problems of the dog (as defined and recognized by the owner) was 1.397 times higher (Fig. 5e; $P<0.05$ ). Moreover, for a oneunit increase in the impaired quality of life index of the owner, the odds ratio for relinquishment was 1.762 times higher (Fig. 5d; $P<0.05)$. Overall, the number of people that recognized behavioral problems in their dogs was low $(11.6 \%$ of the dog owners), as well as the number of people that considered relinquishing their dog (1\%). Still, according to these data, severely impaired quality of life of owners under the COVID-19 pandemic and lockdown was a significant risk factor associated with the quality of life of the dog, as well as for the recognition of the dog's behavioral problems, and for dog relinquishment, as reported by dog owners. Characteristics of the dogs and owners, as well as ownership habits, were controlled in the statistical models, as fully detailed in the "Methods" section and Supplementary Table S3. Further questions that were included in the model regarding the type of behavioral changes in the dogs are presented in Supplementary Table S4.

\section{Methods}

The study was conducted in accordance with the ethical guidelines of The Hebrew University of Jerusalem. As detailed below, data analyzed included four main datasets: (1) retrospective data from the pet adoption website Yad4 (http://Yad4.co.il), an online search engine for adoptable pets in Israel, from January 2016 to May 2020; (2) retrospective data regarding worldwide Google searches for adoptable dogs, downloaded from Google Trends, from November 2016 to May 2020; (3) data gathered from a prospective online digital questionnaire targeting dog owners in Israel, which was active from March 27th, 2020 to April 30th, 2020, during the COVID-19 related full lockdown in Israel; and (4) data gathered from an online digital questionnaire targeting people in Israel who adopted a dog from a shelter during the COVID-19 pandemic; the questionnaire was active from May 20th to May 25th, 2020, a period of time following the gradual opening of the lockdown.

Collection of data regarding abandoned adoptable dogs, adoptions and adopters from Yad4 website. Information was gathered regarding abandoned adoptable dogs, adoptions, and adopter's data, as recorded by Yad4, an open-source online website. In this Israeli website, animal welfare organizations and municipal veterinarians upload individual information for each abandoned dog, typically as soon as it enters the shelter, and this information is available to potential new dog owners who can fill out an online adoption request form through the website to be considered and approved by the shelter. Dataset included: records of the dogs uploaded to the website, date of marking the dog as adopted if indeed adopted, the number of adoption requests sent through the website, as well as requests to serve as foster families. Data regarding the online use of the Yad4 website were extracted from Google Analytics. The database of the Yad4 website was analyzed from January 2016 to May 2020, and included: 33,883 adoptable dogs, 2,618,190 online visits on the website, 53,923 online adoption requests, and 2042 fostering applications. As demonstrated in Fig. 1, data were compared in five different periods: from January 2016 until the outbreak in China; from the initial outbreak in China until the first diagnosed COVID-19 patient in Israel; during the outbreak in Israel, from the diagnosis of the first patient until the full lockdown declared by the Israeli government; during the full lockdown for a month; and during the gradual opening in May 2020.

Collection of data regarding worldwide Google searches for adoptable dogs. Data regarding the use of the website and worldwide Google searches for adoptable dogs were extracted by Google Analytics (https://analytics.google.com). Retrospective data (from January 2016 until May 2020) regarding trends of searches online for "adopt a dog", both in the USA and worldwide, were downloaded from Google Trends (https://trends. google.com) and used for the analysis. As detailed below, data were compared in four different periods: before the COVID-19 outbreak in China; from the media reports about the outbreak in China on December 27th, 2020 until March 13th, 2020-when the World Health Organization (WHO) announced Europe as the epicenter of the pandemic; from the WHO announcement until the gradual opening in May 2020 (the main lockdown in many countries worldwide); and (4) during May 2020 (the gradual opening in many countries).

Online digital questionnaire for dog owners during the COVID-19 related lockdown in Israel. An online digital questionnaire targeting dog owners in Israel was active during the COVID-19 related full lockdown in Israel. The questionnaire was designed by the researchers using Google Forms, and was distributed by a company who specializes in this purpose (Lead Marketing Ltd.; https://leadmarketingltd.com), in order to specifically and effectively reach dog owners in Israel. The online distribution of the questionnaire was based on targeting a predefined group of respondents, with a high level of accuracy, characterized by their interests and online behavior (e.g., users that shop online for dog food or who perform searches for information on dog care). Various digital platforms were used (i.e., Google Display Network, Facebook, Instagram, and others), and banner ads led users to the questionnaire, asking them to voluntarily and anonymously participate in the survey with their consent to be part of this research study.

The questionnaire for dog owners was in Hebrew, and participants were asked to reply to questions regarding their own well-being, as well as the well-being of their companion dog. It included questions regarding the characteristics of the dog population; the source of their dog (e.g., adopted from a shelter, backyard breeding, official breeders), the age of the dog and reproductive status (sterilized or intact), the number of years they have owned the dog, where the dog is kept (e.g., inside an apartment, in a private house, in a garden, free roam). The characteristics of the owners included age, the geographical area in Israel, type of residential area (e.g., city, countryside), and gender. In addition, owners were asked questions regarding their well-being under the lockdown due to the COVID-19 pandemic (on a scale of 1-5); "how stressed are you overall from the COVID-19 pandemic?" (1-not stressed, 5-severely stressed); "to what extent are you worried about your health risk from the COVID-19 epidemic?" (1-not worried, 5-extremely worried); "to what extent the current crisis was harmful to your personal financial income?" (1-not at all, 5-severely harmful); "to what extent was your daily routine altered during this time?" (1-no change, 5-extreme change). In addition, owners were asked how many times a day they walked the dog during the lockdown, the average length of the walk, whether the attention they gave the dog changed (increased, was not changed, or decreased), their assessment of the overall quality of life of their dog under the lockdown (1-remarkedly impaired, 5-markedly improved), if 
there were new behaviors expressed by their dog, and whether or not they were considering relinquishing their dog. For the purpose of analyses regarding the link between human well-being and their answers regarding their dog, an impaired quality of life index was generated, by calculating the average score of the owners based on the responses regarding their overall stress, health concerns, and their personal financial harm due to the COVID-19 epidemic and lockdown, as detailed above. The questionnaire was conducted from March 27th to April 30th, 2020, during the COVID-19 related full lockdown in Israel, and was successfully answered by 3138 individuals. Records were not included if they were incomplete, were completed by people who stated they did not own a dog during the time of the questionnaire or by minors (under 18 years old), or if the age of the dog or the number of years that they raised it were irrational (e.g., 51, 139). Thus, 2906 records were included in the analyses. Characteristics of the participants and their dogs are detailed in Supplementary Fig. S2.

Online digital questionnaire for people in Israel who adopted a dog from a shelter during the COVID-19 pandemic. An online digital questionnaire targeting mainly people in Israel who adopted a dog from a shelter during the COVID-19 pandemic, was active from May 20th to May 25th, 2020, after the gradual opening of the lockdown. The questionnaire was designed by the researchers in Hebrew using Google Forms, and was distributed by Lead Marketing Ltd., in a similar manner as the first questionnaire, in order to effectively target individuals of the predefined group, such as individuals who visited dog adoption websites, mainly Yad4, dog shelters and their Facebook fan pages. Participants were asked to reply to questions regarding the date of dog adoption, the main reason for the specific timing of the adoption (Fig. 5), as well as on the short-term success of the rehoming (e.g., planning to keep the dog, gave it to another family, returned it to the shelter, or considering not keeping it). The questionnaire was answered by 508 participants, and 312 of them stated they adopted the dog during the pandemic (January-May, 2020)

Statistical analyses. Statistical analyses were performed using commercial statistical software (IBM SPSS Statistics, version 24.0; STATA, version 15.0). Linear regression analysis was utilized to evaluate the effects of the spread of COVID-19 and lockdown stages on adoption and abandonment outcomes for pet dogs, using data from the Yad4 adoption website. The general structure of the estimated regressions was as detailed in the Eq. (1).

$$
\begin{gathered}
Y_{t}=\beta_{0}+\delta_{\text {ChinaOutbreak }}+\delta_{\text {LocalOutbreak }}+\delta_{\text {LocalLockown }}+\delta_{\text {OpenUp }}+\gamma_{t} \\
+\beta_{1} \cdot \text { trend }_{t}+\beta_{2} \cdot \text { regulation }_{t}+\varepsilon_{t}
\end{gathered}
$$

where $Y_{t}$ was the outcome of interest in month $t$, the $\delta$ s were dummy variable effects of the stages of outbreak and lockdown, all compared to the baseline period, before the outbreak of COVID-19 in China. $\delta_{\text {ChinaOutbreak }}$ is a dummy variable for the months between the outbreak of COVID-19 in China and the first confirmed case in Israel; $\delta_{\text {LocalOutbreak }}$ is a dummy variable for the period between the first local case and the start of the full lockdown; $\delta_{\text {LocalLockdown }}$-between the start of the lockdown and the start of gradual opening in Israel. $\gamma_{t}$ are the calendar month fixed effects controlling for seasonality in adoption activities, and trend ${ }_{t}$ controls for a linear annual time trend. regulation ${ }_{t}$ controls for a change in governmental initiatives with regard to encouragement of responsible ownership and adoptions between 2018 and 2019 , in order to make sure this does not drive our results. $\varepsilon_{t}$ is a standard error term. Several outcomes were considered variables: the number of adoption requests received through the website and the number of dogs marked as adopted, as measures of the level of interest in conducting an adoption process and the final outcome of successful adoptions; the number of dogs uploaded to the website, as a measure of recent abandonment cases; and the number of users on the website, as a measure of general interest in adoption.

The major part of the analysis uses Israeli data, as detailed above. However, two outcome variables were added from Google Trends to compare the results to worldwide trends. Google Trends were used to construct two additional outcome variables: the number of web-searches, in the US, and worldwide, of the phrase "adopt a dog" during the same period. The regression analysis for these outcomes was similar to the main model, but the stages of shutdown were defined as: the outbreak in China, the declaration of World Health Organization on Europe as the epicenter of the pandemic, and the gradual opening in May 2020. Because the lockdown policies are not centralized in the US and worldwide, we did not include a specific separate shutdown time-period.

The digital questionnaire of the dog owners was analyzed using logistic regression. The binary outcome variables that were considered were: the quality of life of the dog, as assessed by the owner; the development of new behavioral problems if recognized and defined by the owners; and whether the owner was considering abandoning the dog. The general model for estimation was as detailed in Eq. 2.

$$
\begin{aligned}
\operatorname{Prob}\left(Y_{i}=1\right)=\beta_{0} & +\beta_{1} \cdot \text { LifeChange }_{i}+\beta_{2} \cdot \text { CrisisIndex }_{i}+\gamma \cdot Z_{i} \\
& +\delta \cdot W_{i}+\alpha \cdot D_{i}+\nu_{i}+\varepsilon_{i}
\end{aligned}
$$

where $Y_{t}$ is the binary outcome of interest for respondent $\mathrm{i}$. LifeChange $_{i}$ is a dummy variable depicting whether the respondent declared that his life changed following the COVID-19 outbreak. CrisisIndex $x_{i}$ is an average of three responses addressing three aspects of negative effects of the outbreak: economy, health concerns, and stress (as reported by the responders). $Z_{i}$ are owner characteristics: gender, age and whether there are young children in the household. $W_{i}$ are dog characteristics: age, whether the dog was adopted from a shelter, number of years with the owner. $D_{i}$ are characteristics of the care given to the dog: number of walks a day, the average duration of the walks, and a general measure of attention to the dog. $v_{i}$ are geographical area fixed-effects and $\varepsilon_{i}$ is a standard error term. The logistic regressions, as they are based on the responses of the owners, which can be biased on their own, should be interpreted as descriptive analyses rather than being given causal interpretation. Descriptive statistics are given as mean \pm SE, $95 \%$ confidence interval, or as frequency $(n)$ with percentage (\%), as applicable. A $P<0.05$ was considered statistically significant. All reported $P$ values were based on a two-tailed hypothesis.

\section{Discussion}

Humans and dogs are both social animals, and their bond can be traced back at least 15,000 years to the Bonn-Oberkassel dog that was found buried with two humans (Janssens et al., 2016). According to the 2019-2020 National Pet Owners Survey conducted by the American Pet Products Association (APPA), approximately 63.4 million households in the USA owned at least one dog, making them the most widely owned type of companion animal across the USA at this time. The advantages of raising a dog have been widely investigated. The human-dog bond has potential physical, psychological and mental benefits, and can 
improve the general well-being and happiness of owners (LassHennemann et al., 2020; Tzivian et al., 2015; Barker and Barker, 1988; Wells, 2007). Despite all the known advantages, and the evidence that separation between a dog and its owner negatively impacts not only the dog but also the wellness of the owner (Lowe et al., 2015), millions of companion dogs are abandoned every year (Marder and Duxbury, 2008). Dog abandonment carries high costs and a significant risk for public health (Fatjo et al., 2015; Kumar, 2002; Carter, 1990). Prior to this study, it was unknown whether the COVID-19 pandemic was a risk factor for dog abandonment, as well as a risk for impaired well-being of the dogs as a reflection of the potentially impaired well-being of the owners. Therefore, the motivation to conduct this study was to explore the human-dog relationship during this pandemic, to benefit the welfare and well-being of both humans and animals, in accordance with the One Welfare approach. The One Welfare approach extents the One Health theme, suggesting that there is a strong connection between welfare and health of human and animals, including both physical and mental health, and that improving animal welfare often improves human welfare (and vice versa) (Pinillos et al., 2016; Mor et al., 2018; Panning et al., 2016; Lem, 2019; Jordan and Lem, 2014; Card et al., 2018). According to this approach, veterinarians, animal's owners, animal welfare organizations, human psychiatrists, environmental scientists, and others, should collaborate and share expertize in order to care for the welfare of both animals and their owners. Accordingly, the rationale behind this study was the hypotheses that human perceptions and acts regarding dog ownership and adoption might be influenced by the COVID-19 pandemic and the related social isolation, as well as the stress and well-being of both species.

Our data indicate that not only is the concern of increased dog abandonment not justified, at least so far, the opposite has occurred. As social restrictions increased during the COVID-19 pandemic, the rates of dog adoptions improved significantly (Fig. 2); the demand for adoptable dogs and the requests to serve as foster families increased significantly, and accordingly, the length of stay of dogs at the shelter was significantly shorter. Previous reports following disasters, such as earthquakes or other situations that require immediate evacuation, were associated with a massive unintentional dog abandonment (Nagasawa et al., 2012). However, people may refuse to separate from their pet when needed due to disasters or extreme situations, as pet owners may find their pets closer or at the very least, as close as family (Chadwin, 2017; Barker and Barker, 1988). This may be the reason why, so far, the vast majority of people were reluctant to relinquish their dog during the COVID-19 pandemic. Still, further investigation is required, as the potential risk for dog relinquishment in the coming months cannot be completely excluded, due to the various social and economic impacts that this pandemic may yet bring. Furthermore, as our climate continues to change, more disasters, including additional pandemics, will likely occur, highlighting the need for more research into crisis-driven human behavior changes, including changes in the human-animal relationship.

While it may be clear why people kept their companion animals, the motivation to acquire a new dog through adoption, particularly during the COVID-19 related lockdown, is less intuitive. As expected, many people stated they decided to adopt a dog since they had been planning to adopt prior to the COVID-19 outbreak, as well as the fact that people were at home and more available to the new challenge. In addition, acknowledgment of the fact that a dog can reduce feelings of stress and loneliness, as well as misleading media publications about increased dog abandonment, played an important role in their decision. Surprisingly, neither pressure from children and the desire to keep children occupied, nor an excuse to leave the house during the lockdown, were reported to play an important role in the decision of owners to adopt a dog under the circumstances. In the scientific literature, the characteristics of individuals associated with a higher likelihood to adopt a dog, such as ethnicity and housing, were described (Holland, 2019; Weiss et al., 2012); however, the specific timing for adoption has not been investigated. Nevertheless, a previous study found that owners who just obtained a dog expected that the new dog ownership would increase their walking activity, happiness, companionship, and would decrease stress and loneliness (Powell et al., 2018). This may explain the increase in adoption rate during the COVID-19 pandemic, as social isolation was legally enforced.

In addition, to determining the adoption and relinquishment rates associated with COVID-19, we investigated the effect of the stressful pandemic on dog welfare in the pet home environment. Therefore, the questionnaire for dog owners examined the relationship between their impaired quality of life during the pandemic and the quality of life of their companion dog. Although there is an obvious limitation in this study in that the quality of life of the dog and the development of new behavior problems were based subjectively on the owner's perception rather than objectively, the results are nevertheless valuable; as the perceptions of the owners of their dog's behavior is likely a more important predictor of relinquishment than objective measures. Previous researches have found that new owners of dogs often do not report the same behavioral problems as relinquishing owners of the same dog, suggesting that perception plays an important role (Duffy et al., 2014; Stephen and Ledger, 2007).

It was found that impaired quality of life of the owners was associated with a decrease in quality of life of their dog, as well as increased development of new behavioral problems, as judged by their owners. Although it has been reported that owners have poor ability to recognize behavioral problems (Powell et al., 2018; Tami and Gallagher, 2009), the perception of the owners can influence the future of the owner-pet relationship, as well as the probability that they would decide to relinquish it (Payne et al., 2015). Thus, the perception of the owner that their dog has behavioral problems may influence their ownership, and by that, also the welfare of the dog. As mentioned, the quality of life of the dog, and its behavior, were neither diagnosed objectively, nor by professional observers. Therefore, the characteristics of the dogs and of the owners as risk factors for the low quality of life and new behavioral problems of the dog, cannot be thoroughly concluded from these models. Still, those variables were controlled in the statistical models, and it was found that the perception of dog owners regarding their own impaired quality of life was significantly associated with their assessment of lower quality of their dog's life and its emerging behavioral problems. These results are consistent with previous studies, which found that there is an effect of the stress level and well-being of the humans, on the stress, well-being, cognitive ability and behavior of their dogs (Buttner et al., 2015; Sumegi et al., 2014; Kaminski et al., 2009). An alternative hypothesis may be that owners in a crisis situation may have had a pessimistic outlook regarding things in their life and their surroundings, and the resulting decrease of the quality of life of the dog was due to the overall negative outlook of the owner rather than any true reflection on the dog. This information is important for both humans and dogs, since it can provide valuable information for initiatives to improve the welfare and well-being of both the dog owners and their companion dogs, as suggested by the One Welfare approach. For example, the Israeli Veterinary Services (a branch of the Ministry of Agriculture), invests an annual amount of approximately $\$ 1.2 \mathrm{M}$ on encouraging responsible dog ownership and adoptions, and has already approved new upcoming initiatives based on this study, such as digital online adoption days, with education geared towards responsible dog ownership. 
In this study, although the overall number of dog owners who reported that they were going to relinquish their dog due to the COVID-19 situation was low, it was significantly associated with a poorer quality of life index of the owners. Among new dog owners that had adopted the dog during the pandemic, a similar percentage is reported for owners that had already relinquished their dog or were considering doing so. Since a lack of time of the owners is one of the main risk factors for dog relinquishment reported in the literature (Salman et al., 2000), it was a concern that people who adopted during the COVID-19 lockdown would relinquish their dog after going back to routine life. The second survey to detect the relinquishment rate of dogs adopted during the pandemic was performed at the end of May 2020, after opening the lockdown. Therefore, in most cases, people were back to routine life more than a month following adoption. According to previous research, the highest proportion of dog relinquishment happens just one month after adoption; in fact, owners report knowing of behavioral problems with their dogs within $24 \mathrm{~h}$ post-adoption (Shore, 2005). It is important to mention that people who decided to relinquish their dog might have avoided our survey. Still, the relatively low number of dogs that were uploaded to Yad4 website on May 2020 may indicate that, so far, there has not been a massive relinquishment after opening the lockdown. Hence, we tentatively suggest that the majority of adoptions were successful. One hypothesis can be that owners had more time to spend with their dog at the beginning, which may have helped to ease rehoming. Nonetheless, this hypothesis requires further investigation in the long run. Furthermore, an important issue that was not covered in this study is related to the differences between individuals who had already owned a dog and those who had not, in regards to their coping with the extreme social and economic challenges during the COVID-19 pandemic. Still, studies show that both children and adults cope better with stress when owning a dog (Chadwin, 2017; Powell et al., 2019). Therefore, we hypothesize that owning a dog might even prevent the development of Post Traumatic Stress Disorders (PTSD) caused by the pandemic or at least ease the coping with it, once it has occurred. It has been reported that after the SARS outbreak in 2003, which may be equivalent to the COVID-19 pandemic in many aspects, patients suffered from Post Traumatic Stress Disorders (Wu et al., 2005). It is known that dogs have a positive effect on the treatment of PTSD, and that dog owners might be more resilient (Chadwin, 2017; Powell et al., 2019; Beetz et al., 2019). Therefore, this is an important future direction for human-pet relationship research.

In summary, the COVID-19 pandemic that emerged in December 2019 in Wuhan, China, led to the utilization of social isolation in many countries, as well as to widespread uncertainty and severe health and economic concerns. Our study indicates that the stricter the social isolation became during the COVID-19 pandemic, the greater the interest in dog adoption. The adoption rate increased significantly, while dog abandonment did not change. Furthermore, there was a clear association between individual's quality of life and their perceptions of their dog's quality of life and behavior, as well as the probability of relinquishing the dog. As humans and dogs are both social animals, these findings suggest potential benefits of the human-dog relationships during the COVID-19 pandemic, in accordance with the One Welfare approach implying that there is a bidirectional connection between the welfare and health of humans and nonhuman animals.

\section{Data availability}

All data generated or analyzed during this study is included in this article (and its Supplementary Information files).
Received: 6 July 2020; Accepted: 29 October 2020;

Published online: 24 November 2020

\section{References}

Anser MK, Yousaf Z, Khan MA, Nassani AA, Alotaibi SM, Qazi Abro MM, Vo XV, Zaman K (2020) Does communicable diseases (including COVID-19) may increase global poverty risk? A cloud on the horizon. Environ Res 187:109668

Barker SB, Barker RT (1988) The human-canine bond: closer than family ties? J Mental Health Counsel 10(1):46-56

Bavel JJV, Baicker K, Boggio PS, Capraro V, Cichocka A, Cikara M, Crockett MJ Crum AJ, Douglas KM, Druckman JN, Drury J, Dube O, Ellemers N, Finkel EJ, Fowler JH, Gelfand M, Han S, Haslam SA, Jetten J, Kitayama S, Mobbs D, Napper LE, Packer DJ, Pennycook G, Peters E, Petty RE, Rand DG, Reicher SD, Schnall S, Shariff A, Skitka LJ, Smith SS, Sunstein CR, Tabri N, Tucker JA, Linden SVD, Lange PV, Weeden KA, Wohl MJA, Zaki J, Zion SR, Willer $R$ (2020) Using social and behavioural science to support COVID-19 pandemic response. Nat Human Behav 4(5):460-471

Beetz A, Schofmann I, Girgensohn R, Braas R, Ernst C (2019) Positive effects of a short-term dog-assisted intervention for soldiers with post-traumatic stress disorder-a pilot study. Front Vet Sci 6:170

Beetz A, Uvnas-Moberg K, Julius H, Kotrschal K (2012) Psychosocial and psychophysiological effects of human-animal interactions: the possible role of oxytocin. Front Psychol 3:234

Bojdani E, Rajagopalan A, Chen A, Gearin P, Olcott W, Shankar V, Cloutier A, Solomon H, Naqvi NZ, Batty N, Festin FED, Tahera D, Chang G, DeLisi LE (2020) COVID-19 pandemic: impact on psychiatric care in the United States. Psychiatry Res 289:113069

Burgos-Caceres S (2011) Canine rabies: a looming threat to public health. Animals $1(4): 326-342$

Buttner AP, Thompson B, Strasser R, Santo J (2015) Evidence for a synchronization of hormonal states between humans and dogs during competition. Physiol Behav 147:54-62

Card C, Epp T, Lem M (2018) Exploring the social determinants of animal health. J Vet Med Educ 45(4):437-447

Carter CN (1990) Pet population control: another decade without solutions? J Am Vet Med Assoc 197(2):192-195

Chadwin R (2017) Evacuation of pets during disasters: a public health intervention to increase resilience. Am J Public Health 107(9):1413-1417

Duffy DL, Kruger KA, Serpell JA (2014) Evaluation of a behavioral assessment tool for dogs relinquished to shelters. Prev Vet Med 117(3-4):601-609

Fatjo J, Bowen J, Garcia E, Calvo P, Rueda S, Amblas S, Lalanza JF (2015) Epidemiology of dog and cat abandonment in Spain (2008-2013). Animals 5 (2):426-441

Goumenou M, Spandidos DA, Tsatsakis A (2020) [Editorial] Possibility of transmission through dogs being a contributing factor to the extreme Covid19 outbreak in North Italy. Mol Med Rep 21(6):2293-2295

Holland KE (2019) Acquiring a pet dog: a review of factors affecting the decisionmaking of prospective dog owners. Animals 9(4):124

Janssens LA, Street M, Miller R, Hazewinkel HA, Giemsch L, Schmitz R (2016) The oldest case yet reported of osteoarthritis in a dog: an archaeological and radiological evaluation. J Small Anim Pract 57(10):568-574

Jordan T, Lem M (2014) One health, one welfare: education in practice veterinary students' experiences with community veterinary outreach. Can Vet J 55 (12):1203-1206

Kaminski J, Brauer J, Call J, Tomasello M (2009) Domestic dogs are sensitive to a human's perspective. Behaviour 146:979-998

Koo JR, Cook AR, Park M, Sun Y, Sun H, Lim JT, Tam C, Dickens BL (2020) Interventions to mitigate early spread of SARS-CoV-2 in Singapore: a modelling study. Lancet Infect Dis 20(6):678-688

Kumar S (2002) Stray dogs are a growing threat to public health. BMJ 325 (7355):66-66

Lass-Hennemann J, Schafer SK, Sopp MR, Michael T (2020) The relationship between dog ownership, psychopathological symptoms and healthbenefitting factors in occupations at risk for traumatization. Int $J$ Environ Res Public Health 17(7):2562

LeDoux J (2012) Rethinking the emotional brain. Neuron 73(4):653-676

Lem M (2019) Serving homeless populations through a One Health approach. Can Vet J 60(10):1119-1120

Leroy EM, Ar Gouilh M, Brugere-Picoux J (2020) The risk of SARS-CoV-2 transmission to pets and other wild and domestic animals strongly mandates a one-health strategy to control the COVID-19 pandemic. One Health 10:100133

Lewnard JA, Lo NC (2020) Scientific and ethical basis for social-distancing interventions against COVID-19. Lancet Infect Dis 20(6):631-633

Lowe SR, Joshi S, Pietrzak RH, Galea S, Cerda M (2015) Mental health and general wellness in the aftermath of Hurricane Ike. Soc Sci Med 124:162-170 
Marder A, Duxbury MM (2008) Obtaining a pet: realistic expectations. Vet Clin North Am Small Anim Pract 38(5):1145-1162

Mobbs D, Hagan CC, Dalgleish T, Silston B, Prevost C (2015) The ecology of human fear: survival optimization and the nervous system. Front Neurosci 9:55

Mor SM, Norris JM, Bosward KL, Toribio J, Ward MP, Gongora J, Vost M, Higgins PC, McGreevy PD, White PJ, Zaki S (2018) One health in our backyard: Design and evaluation of an experiential learning experience for veterinary medical students. One Health 5:57-64

Nagasawa M, Mogi K, Kikusui T (2012) Continued distress among abandoned dogs in Fukushima. Sci Rep 2:724

Panning C, Lem M, Bateman S (2016) Profiling a one-health model for priority populations. Can J Public Health 107(3):e222-e223

Parry NMA (2020) COVID-19 and pets: when pandemic meets panic. Forensic Sci Int 2:100090-100090

Patronek GJ, Glickman LT, Beck AM, McCabe GP (1996) Risk factors for relinquishment of dogs to an animal shelter. J Am Vet Med Assoc 209(3):572-581

Payne E, Bennett PC, McGreevy PD (2015) Current perspectives on attachment and bonding in the dog-human dyad. Psychol Res Behav Manag 8:71-79

Payne E, DeAraugo J, Bennett P, McGreevy P (2016) Exploring the existence and potential underpinnings of dog-human and horse-human attachment bonds. Behav Proces 125:114-121

Pinillos RG, Appleby MC, Manteca X, Scott-Park F, Smith C, Velarde A (2016) One welfare-a platform for improving human and animal welfare. Vet Rec 179(16):412-413

Powell L, Chia D, McGreevy P, Podberscek AL, Edwards KM, Neilly B, Guastella AJ, Lee V, Stamatakis E (2018) Expectations for dog ownership: Perceived physical, mental and psychosocial health consequences among prospective adopters. PLoS ONE 13(7):e0200276

Powell L, Edwards KM, McGreevy P, Bauman A, Podberscek A, Neilly B, Sherrington C, Stamatakis E (2019) Companion dog acquisition and mental well-being: a community-based three-arm controlled study. BMC Public Health 19(1):1428

Ryan MG, Storey AE, Anderson RE, Walsh CJ (2019) Physiological indicators of attachment in domestic dogs (Canis familiaris) and their owners in the strange situation test. Front Behav Neurosci 13:162

Salman MD, Hutchison J, Ruch-Gallie R, Kogan L, New JC, Kass PH, Scarlett JM (2000) Behavioral reasons for relinquishment of dogs and cats to 12 shelters. J Appl Animal Welfare Sci 3(2):93-106

Sangar S, Dutt V, Thakur R (2019) Comparative assessment of economic burden of disease in relation to out of pocket expenditure. Front Public Health 7:9

Serpell J (1991) Beneficial effects of pet ownership on some aspects of human health and behaviour. J R Soc Med 84(12):717-720

Shore ER (2005) Returning a recently adopted companion animal: adopters' reasons for and reactions to the failed adoption experience. J Appl Anim Welf Sci 8(3):187-198

Stephen J, Ledger R (2007) Relinquishing dog owners' ability to predict behavioural problems in shelter dogs post adoption. Appl Animal Behav Sci 107(1):88-99

Sumegi Z, Olah K, Topal J (2014) Emotional contagion in dogs as measured by change in cognitive task performance. Appl Animal Behav Sci 160:106-115

Tami G, Gallagher A (2009) Description of the behaviour of domestic dog (Canis familiaris) by experienced and inexperienced people. Appl Animal Behav Sci 120(3):159-169

Tzivian L, Friger M, Kushnir T (2015) Associations between stress and quality of life: differences between owners keeping a living dog or losing a dog by euthanasia. PLoS ONE 10(3):e0121081

Weiss E, Miller K, Mohan-Gibbons H, Vela C (2012) Why did you choose this pet?: Adopters and pet selection preferences in five animal shelters in the United States. Animals 2(2):144-159

Wells DL (2007) Domestic dogs and human health: an overview. Br J Health Psychol 12(Pt 1):145-156
Wu KK, Chan SK, Ma TM (2005) Posttraumatic stress after SARS. Emerg Infect Dis 11(8):1297-1300

Xiao H, Zhang Y, Kong D, Li S, Yang N (2020) Social capital and sleep quality in individuals who self-isolated for 14 days during the Coronavirus Disease 2019 (COVID-19) outbreak in January 2020 in China. Med Sci Monit 26:e923921

\section{Acknowledgements}

We thank the Yad4.co.il website for sharing their data, and specifically to the technical developer of YAD4, Mr. Omri Amos, for extracting the data, as well as the CTS group, and Farmina pet-food, Israel, the current supporters of the website. We thank all study participants and adopters who were willing to provide information. We thank the Universities Federation for Animal Welfare (UFAW) for their support in all our work regarding animal welfare in Israel. Gratitude to Ms. Odelya Natan and her team from Lead Marketing Ltd.

\section{Author contributions}

T.R. supervised the project, conceived the ideas and study design, and revised the manuscript. B.Y. co-supervisor of the project. L.M. conceived the ideas, conducted the study, analyzed the results, and wrote the first draft of the manuscript. A.P. took part in the study design, ideas, and writing of the manuscript. R.B. assisted with the analyses and revised the manuscript. B.I.S contributed with the ideas, design, conducted the statistical analyses, and participated in writing the manuscript. G.S. conducted statistical anlyses. A G. collected data. All authors read, revised, and approved the manuscript.

\section{Competing interests}

L.M is the founder and the owner of Yad4 website. The ownership of the website had no influence on the objectivity and the integrity of the manuscript. The remaining authors declare no competing interests.

\section{Additional information}

Supplementary information is available for this paper at https://doi.org/10.1057/s41599020-00649-x.

Correspondence and requests for materials should be addressed to T.R.

Reprints and permission information is available at http://www.nature.com/reprints

Publisher's note Springer Nature remains neutral with regard to jurisdictional claims in published maps and institutional affiliations.

Open Access This article is licensed under a Creative Commons Attribution 4.0 International License, which permits use, sharing, adaptation, distribution and reproduction in any medium or format, as long as you give appropriate credit to the original author(s) and the source, provide a link to the Creative Commons license, and indicate if changes were made. The images or other third party material in this article are included in the article's Creative Commons license, unless indicated otherwise in a credit line to the material. If material is not included in the article's Creative Commons license and your intended use is not permitted by statutory regulation or exceeds the permitted use, you will need to obtain permission directly from the copyright holder. To view a copy of this license, visit http://creativecommons.org/ licenses/by/4.0/

(C) The Author(s) 2020

\section{Liat Morgan (10), Alexandra Protopopova², Rune Isak Dupont Birkler ${ }^{3}$, Beata Itin-Shwartz ${ }^{4}$, Gila Abells Sutton?, Alexandra Gamliel', Boris Yakobson ${ }^{5} \&$ Tal Raz (iD $^{1 \times}$}

${ }^{1}$ Koret School of Veterinary Medicine, Robert H. Smith Faculty of Agriculture, Food and Environment, The Hebrew University of Jerusalem, Jerusalem, Israel. ${ }^{2}$ Faculty of Land and Food Systems, University of British Columbia, Vancouver, BC V6T 1Z4, Canada. ${ }^{3}$ BLAVATNIK CENTER for Drug Discovery, Metabolite Medicine Division, Tel Aviv University, Tel Aviv, Israel. ${ }^{4}$ Environmental Economics and Management, Robert H. Smith Faculty of Agriculture, Food and Environment, The Hebrew University of Jerusalem, Jerusalem, Israel. ${ }^{5}$ Ministry of Agriculture and Rural

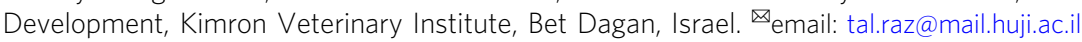

